\title{
Can the Onset of Atrophic Age-Related Macular Degeneration Be an Acceptable Endpoint for Preventative Trials?
}

\author{
Zhichao Wua, Robyn H. Guymer ${ }^{a, b}$ \\ ${ }^{a}$ Centre for Eye Research Australia, Royal Victorian Eye and Ear Hospital, East Melbourne, VIC, Australia; \\ bophthalmology, Department of Surgery, The University of Melbourne, Melbourne, VIC, Australia
}

\section{Keywords}

Age-related macular degeneration · Geographic atrophy · Endpoints · Trials

\begin{abstract}
The slowly progressive nature of age-related macular degeneration (AMD) means that establishing the efficacy of novel preventative treatments aiming to slow progression of disease, remains challenging, and where earlier endpoints are needed to improve their feasibility. This review examines whether the onset of atrophic AMD, as seen as anatomical signs on optical coherence tomography termed nascent geographic atrophy, could act as a useful surrogate endpoint for early intervention trials.

๑) 2020 S. Karger AG, Basel
\end{abstract}

\section{Introduction}

Age-related macular degeneration (AMD) remains the leading cause of irreversible vision loss, with nearly 200 million individuals worldwide estimated to have AMD in 2020 [1]. Amongst these individuals, approximately 8 million people are estimated to have late AMD, including atrophic or neovascular AMD (nAMD).
Those who develop atrophic AMD face an inevitable future of progressive vision loss as a result of the degeneration of the retinal layers responsible for vision due to the lack of effective treatments to slow or prevent its progression. Whilst anti-vascular endothelial growth factor (VEGF) treatments are now available and highly effective at treating the neovascular complications of AMD, less than half of those undergoing treatment retain a VA of $20 / 40$ or better after 5 years [2]. Continued vision loss, despite adequate treatment, often occurs as a result of the onset of atrophy.

Preventative treatments to effectively slow or delay the progression to late AMD in the early stages of the disease are thus urgently needed to prevent irreversible vision loss. However, the slowly progressive nature of AMD means that large and lengthy trials are required to detect clinically meaningful treatment effects for preventative treatment trials that start early in the disease process. This review therefore explores whether alternative, earlier endpoints, specifically related to the onset of atrophic AMD, could be acceptable as this would greatly improve the feasibility of conducting early intervention trials aimed at slowing progression to vison threatening late complications.

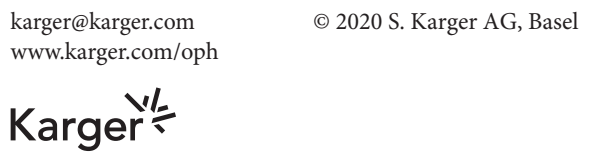




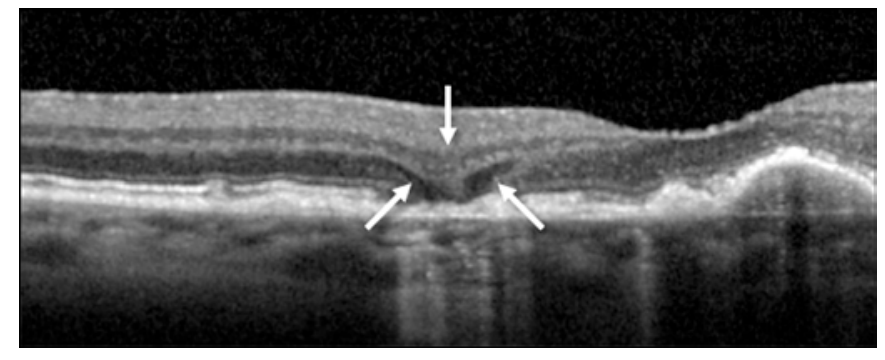

Fig. 1. OCT B-scan through an area with nascent geographic atrophy (nGA), evident by the subsidence of the INL and OPL (vertical arrow) and hyporeflective wedge-shaped bands within Henle's fiber layer (diagonal arrows), both indicating a loss of the photoreceptors.

\section{Endpoints Used in Previous Preventative Treatment Trials}

The primary endpoints used to date in several large preventative treatment trials [3-6] have included the development of $\mathrm{nAMD}$ on color fundus photographs (CFPs) with or without fluorescein angiography, geographic atrophy (GA) on CFPs, or visual acuity (VA). VA outcome remains an important endpoint from a regulatory perspective [7], but there are several important confounders that affect the suitability of this outcome measure. For instance, VA is dependent on the degree of delay to presentation when nAMD develops, as well as one's response to treatment with anti-VEGF [8]. When GA develops, it is often outside the foveal region [9], meaning that VA outcomes fail to capture disease progression in eyes that develop non-foveal GA. In both instances, disease progression to late AMD is poorly captured by VA and as such this outcome is not a useful endpoint in preventative trials aiming to slow progression from early disease with good vision to sight-threatening late AMD where VA can remain unchanged.

\section{Onset of Atrophic AMD}

Optical coherence tomography (OCT) imaging has enabled us to observe the early signs of atrophy that are not clinically visible nor detectable on CFPs. These OCT signs, indicating the onset of the atrophic AMD process, were originally termed nascent geographic atrophy (nGA) [10]. In a large cohort of intermediate AMD cases, all of whom had bilateral large drusen, with or without pigmentary changes, these early signs of atrophic AMD were observed to be present in one in eight eyes [11]. More recently, the term incomplete retinal pigment epithelial and outer retinal atrophy (iRORA) has been used to include a broadened range of early anatomical changes associated with the atrophic process, and the term nGA has been suggested to be used to include these changes [12]. However, the discussion here is based upon the original definition of nGA, requiring the presence of one or both of following two features: (i) subsidence of the inner nuclear layer (INL) and outer plexiform layer (OPL) and/or, (ii) a hyporeflective wedge-shaped bands within Henle's fiber layer as evidence of photoreceptor loss (example shown in Fig. 1).

Areas of nGA have been observed to predominantly be characterized by hyper- and hypoautofluorescence on fundus autofluorescence (FAF) imaging (indicating abnormalities at the retinal pigment epithelium and photoreceptor level [13]) and also choriocapillaris alterations on OCT angiography imaging [14]. Visual sensitivity as measured using microperimetry is also on average lower over these regions of nGA than over other non-atrophic features in the early stages of AMD, but are not yet areas of absolute scotomas that typically characterize areas of GA [15].

A previous study demonstrated that eyes detected as having nGA at baseline were associated with a 56-fold increased risk of developing GA over an average follow-up period of 9 years [16]. More recently, we demonstrated that amongst individuals with bilateral large drusen that developed nGA as detected on 6-monthly follow-up intervals for 3 years, there was a 78 -fold increased rate of progression to GA as compared to eyes that did not develop nGA [17]. In this cohort, the probability of a person with bilateral large drusen developing nGA in either eye over a 36 -month period was $24 \%$, being substantially higher than a $13 \%$ probability of developing GA alone. Therefore, the sample size needed for trials using a combined atrophic endpoint of both nGA and GA would be substantially reduced compared to a trial relying on conventional GA alone.

This accumulating body of evidence collectively highlights how nGA has been observed to precede and be strongly associated with an increased risk of developing of GA but is characterized by less severe structural and functional changes associated with frank GA. As such, preventing the development of nGA would likely indicate the prevention of photoreceptor loss, which has been considered to be a clinically meaningful endpoint [7]. In 2017, the United States Food and Drug Administration (FDA) suggested that preventing photoreceptor loss 
would be considered a clinically meaningful trial endpoint given the established link between photoreceptor loss and visual function [7]. This collectively suggests how nGA may potentially be a useful surrogate endpoint in preventative treatment trials.

\section{Assessment of Surrogate Endpoints and Challenges}

In considering surrogate endpoints, Fleming and DeMets [18] emphasized, a significant correlation between a variable and the clinical endpoint is not sufficient to establish its utility as a surrogate endpoint. Instead, a potential surrogate also needs to satisfy four operational criteria for a useful surrogate endpoint as proposed by Prentice [19]. In the context of assessing whether nGA would be a useful surrogate for the conventional clinical endpoint of GA, these criteria include: (1) a significant effect of treatment on the surrogate (nGA); (2) a significant effect of treatment on the clinical endpoint (GA); (3) a significant correlation between the surrogate and clinical endpoint; and (4) the effect of treatment on the clinical endpoint becomes non-significant after accounting for the surrogate endpoint, indicating that the effect of treatment on the clinical endpoint can be captured by the surrogate.

The significant association between the surrogate and clinical endpoint alone (Prentice's Criterion III) is insufficient to justify the validity of a surrogate because it is possible for the disease process to affect the clinical outcome through a casual pathway not involving the surrogate. In atrophic AMD for example, it is possible for GA to develop through a pathway not mediated by nGA (such as by the result of outer retinal atrophy associated with reticular pseudodrusen [20]).

It is also possible for the treatment to only have an effect on disease pathways that involve the surrogate (satisfying Prentice's Criterion I), if for example a treatment was only effective for slowing progression for eyes that first develop nGA before GA, but not for eyes that develop GA without nGA being first present. Similarly, it is possible for the treatment to only have an effect on the clinical endpoint (satisfying Prentice's Criterion II) and not the surrogate endpoint, such as if a treatment slows progression to GA, but not necessarily nGA. It is also possible for treatments to have an effect on the clinical endpoint through a mechanism that is entirely independent of the disease process [18]. Therefore, the criteria set by Prentice [19] requires that the effect of treatment on the clinical endpoint to be captured by the surrogate end-

Atrophic AMD Onset in Preventative

Trials point, rendering the statistically significant effect of treatment on the clinical endpoint to a position of non-significance after accounting for the surrogate (Prentice's Criterion IV). However, a useful surrogate does not need to perfectly capture the effect of treatment on the clinical endpoint [21]. As such, it has been previously argued that calculating the proportion of treatment effect explained (PTE) by the surrogate endpoint would be a useful method for further validating Prentice's Criterion IV [21,22], but many studies have also described important limitations with this method [21, 23-25].

As can be noted by the above requirements, validation of a surrogate endpoint requires the availability of an effective preventative treatment demonstrating strong efficacy, which remains challenging in AMD where the lack of the surrogate endpoint is a major impediment for the conduct of preventative treatment trials. This vicious circle adds to the already challenging task of rigorous statistical validation of surrogate endpoints in general [26].

\section{The Way Forward for Surrogate Endpoints in Preventative Trials}

In view of these challenges, some have suggested that a pragmatic approach may be needed in such scenarios, encouraging the acceptance and adoption of surrogate endpoints on the basis of biological plausibility [27]. This approach would be relevant when considering whether onset of nGA would be an acceptable surrogate endpoint for the conventional endpoint of GA, given its strong biological plausibility based on the evidence available to date. These pragmatic considerations led to a combined atrophic endpoint of late $\mathrm{AMD}$, including $\mathrm{nGA}$, to being used as an endpoint in our recent randomized controlled trial of a novel subthreshold nanosecond laser treatment aiming to slow or prevent disease progression in those with bilateral large drusen with no evidence of nGA at baseline [28]. Further discussions are now needed with regulatory authorities to better understand the acceptability of such an endpoint for approval of new therapeutics.

The acceptance and adoption of nGA (or iRORA) onset as a surrogate endpoint would be further strengthened by establishing whether grading centers can accurately and reproducibly identify the features that indicate cell loss on an OCT. This work is currently underway by the Classification of Atrophy Meetings (CAM) group and establishing the robustness of individual anatomical changes that indicate cell loss may lead to further refinement of 
the definition of nGA (or iRORA) to ensure that it is sufficiently reproducible as a surrogate endpoint that can be used in clinical trials. Any change in the final definition of $\mathrm{nGA}$, as a result of grading reproducibility, would need to ensure that the signs remained strongly associated with the development of the clinical endpoint of GA, since a previous study observed that the different anatomical features that indicate cell loss conferred different risks for GA development [16]. Once this is achieved, planning, conducting and funding future preventative trials will be greatly enhanced. The inclusion of nGA as part of a combined atrophic endpoint with GA will accelerate our identification of the most promising interventions in earlier phase studies and improve the efficiency of larger studies.

\section{Conclusions}

Interventions are urgently needed to prevent irreversible vision loss from AMD, but large and lengthy trials are currently needed to evaluate such treatments when relying on conventional clinical endpoints. Surrogate endpoints have the potential to dramatically improve the feasibility of such trials and thus expedite the discovery of such preventative treatments. However, rigorous statistical validation of a surrogate endpoint remains highly challenging, especially given the requirement for an effec- tive treatment showing strong efficacy. To overcome these challenges, a pragmatic approach of considering the biological plausibility of a potential surrogate endpoint is necessary. The onset of nGA detected on OCT imaging represents such a potential surrogate endpoint exhibiting strong biological plausibility that warrants consideration for acceptance for use in AMD preventative trials once it has been established that its characteristic features can be reproducibly graded.

\section{Conflict of Interest Statement}

R.H.G. reports personal fees from Bayer, Novartis, Roche Genentech and Apellis outside the submitted work and research grant from Bayer outside the submitted work. Z.W. has nothing to declare.

\section{Funding Sources}

This study was supported by the Margaret Miller Foundation (Z.W.).

\section{Author Contributions}

Z.W. and R.H.G. were both responsible for the preparation, review and approval of this study.

\section{References}

1 Wong WL, Su X, Li X, Cheung CM, Klein R, Cheng CY, et al. Global prevalence of age-related macular degeneration and disease burden projection for 2020 and 2040: a systematic review and meta-analysis. Lancet Glob Health. 2014 Feb;2(2):e106-16.

2 Gillies MC, Campain A, Barthelmes D, Simpson JM, Arnold JJ, Guymer RH, et al.; Fight Retinal Blindness Study Group. Long-Term Outcomes of Treatment of Neovascular AgeRelated Macular Degeneration: Data from an Observational Study. Ophthalmology. 2015 Sep;122(9):1837-45.

3 Age-Related Eye Disease Study Research Group. A randomized, placebo-controlled, clinical trial of high-dose supplementation with vitamins $\mathrm{C}$ and $\mathrm{E}$, beta carotene, and zinc for age-related macular degeneration and vision loss: AREDS report no. 8. Arch Ophthalmol. 2001 Oct;119(10):1417-36.
4 Complications of Age-Related Macular Degeneration Prevention Trial Research Group. Laser treatment in patients with bilateral large drusen: the complications of age-related macular degeneration prevention trial. Ophthalmology. 2006 Nov;113(11):1974-86.

5 Friberg TR, Brennen PM, Freeman WR, Musch DC; PTAMD Study Group. Prophylactic treatment of age-related macular degeneration report number 2: 810-nanometer laser to eyes with drusen: bilaterally eligible patients. Ophthalmic Surg Lasers Imaging. 2009 Nov-Dec;40(6):530-8.

6 Age-Related Eye Disease Study 2 Research Group. Lutein + zeaxanthin and omega- 3 fatty acids for age-related macular degeneration: the Age-Related Eye Disease Study 2 (AREDS2) randomized clinical trial. JAMA. 2013 May;309(19):2005-15.

7 Csaky K, Ferris F 3rd, Chew EY, Nair P, Cheetham JK, Duncan JL. Report From the NEI/FDA Endpoints Workshop on Age-Related Macular Degeneration and Inherited Retinal Diseases. Invest Ophthalmol Vis Sci. 2017 Jul;58(9):3456-63.
8 Lim JH, Wickremasinghe SS, Xie J, Chauhan DS, Baird PN, Robman LD, Hageman G, Guymer RH: Delay to treatment and visual outcomes in patients treated with anti-vascular endothelial growth factor for age-related macular degeneration. Am J Ophthalmol. 2012; 153: 678-86. e672. https://doi. org/10.1016/j.ajo.2011.09.013.

9 Sleiman K, Veerappan M, Winter KP, McCall MN, Yiu G, Farsiu S, et al.; Age-Related Eye Disease Study 2 Ancillary Spectral Domain Optical Coherence Tomography Study Group. Optical Coherence Tomography Predictors of Risk for Progression to Non-Neovascular Atrophic Age-Related Macular Degeneration. Ophthalmology. 2017 Dec; 124(12):1764-77.

10 Wu Z, Luu CD, Ayton LN, Goh JK, Lucci LM, Hubbard WC, et al. Optical coherence tomography-defined changes preceding the development of drusen-associated atrophy in age-related macular degeneration. Ophthalmology. 2014 Dec;121(12):2415-22. 
11 Wu Z, Ayton LN, Luu CD, Baird PN, Guymer RH. Reticular Pseudodrusen in Intermediate Age-Related Macular Degeneration: Prevalence, Detection, Clinical, Environmental, and Genetic Associations. Invest Ophthalmol Vis Sci. 2016 Mar;57(3):1310-6.

12 Guymer RH, Rosenfeld PJ, Curcio CA, Holz FG, Staurenghi G, Freund KB, et al. Incomplete Retinal Pigment Epithelial and Outer Retinal Atrophy in Age-Related Macular Degeneration: Classification of Atrophy Meeting Report 4. Ophthalmology. 2020 Mar; 127(3):394-409.

$13 \mathrm{Wu}$ Z, Luu CD, Ayton LN, Goh JK, Lucci LM, Hubbard WC, et al. Fundus autofluorescence characteristics of nascent geographic atrophy in age-related macular degeneration. Invest Ophthalmol Vis Sci. 2015 Feb;56(3):1546-52.

14 Moult EM, Waheed NK, Novais EA, Choi W, Lee B, Ploner SB, et al. Swept-source optical coherence tomography angiography reveals choriocapillaris alterations in eyes with nascent geographic atrophy and drusen-associated geographic atrophy. Retina. 2016 Dec;36 Suppl 1:S2-11.

15 Wu Z, Ayton LN, Luu CD, Guymer RH. Microperimetry of nascent geographic atrophy in age-related macular degeneration. Invest Ophthalmol Vis Sci. 2014 Dec;56(1):115-21.
16 Ferrara D, Silver RE, Louzada RN, Novais EA, Collins GK, Seddon JM. Optical Coherence Tomography Features Preceding the Onset of Advanced Age-Related Macular Degeneration. Invest Ophthalmol Vis Sci. 2017 Jul; 58(9):3519-29.

$17 \mathrm{Wu}$ Z, Luu CD, Hodgson LA, Caruso E, Tindill N, Aung KZ, et al. Prospective Longitudinal Evaluation of Nascent Geographic Atrophy in Age-Related Macular Degeneration. Ophthalmol Retina. 2020 Jun;4(6):568-75.

18 Fleming TR, DeMets DL. Surrogate end points in clinical trials: are we being misled? Ann Intern Med. 1996 Oct;125(7):605-13.

19 Prentice RL. Surrogate endpoints in clinical trials: definition and operational criteria. Stat Med. 1989 Apr;8(4):431-40.

20 Spaide RF. Outer retinal atrophy after regression of subretinal drusenoid deposits as a newly recognized form of late age-related macular degeneration. Retina. 2013 Oct; 33(9):1800-8.

21 Lin DY, Fleming TR, De Gruttola V. Estimating the proportion of treatment effect explained by a surrogate marker. Stat Med. 1997 Jul;16(13):1515-27.

22 Freedman LS, Graubard BI, Schatzkin A. Statistical validation of intermediate endpoints for chronic diseases. Stat Med. 1992 Jan;11(2): $167-78$.
23 Flandre P, Saidi Y. Estimating the proportion of treatment effect explained by a surrogate marker by DY Lin, TR Fleming and V. De Gruttola, Statistics in Medicine, 16, 15151527 (1997). Stat Med. 1999;18(1):107-9.

24 Buyse M, Molenberghs G. Criteria for the validation of surrogate endpoints in randomized experiments. Biometrics. 1998 Sep;54(3): 1014-29.

25 Daniels MJ, Hughes MD. Meta-analysis for the evaluation of potential surrogate markers. Stat Med. 1997 Sep;16(17):1965-82.

26 Amur S, LaVange L, Zineh I, Buckman-Garner S, Woodcock J. Biomarker qualification: toward a multiple stakeholder framework for biomarker development, regulatory acceptance, and utilization. Clin Pharmacol Ther. 2015 Jul;98(1):34-46.

27 Buyse M, Sargent DJ, Grothey A, Matheson A, de Gramont A. Biomarkers and surrogate end points-the challenge of statistical validation. Nat Rev Clin Oncol. 2010 Jun;7(6):309-17.

28 Guymer RH, Wu Z, Hodgson LA, Caruso E, Brassington $\mathrm{KH}$, Tindill N, et al.; Laser Intervention in Early Stages of Age-Related Macular Degeneration Study Group. Subthreshold Nanosecond Laser Intervention in Age-Related Macular Degeneration: The LEAD Randomized Controlled Clinical Trial. Ophthalmology. 2019 Jun;126(6):829-38. 